\title{
Editorial
}

\section{Psychoneuroimmunology Journal_Welcome to a New Journal}

\author{
Ning Quan \\ Charles E. Schmidt College of Medicine, Florida Atlantic University, 5353 Parkside Drive, Jupiter, FL 33458, USA \\ Address correspondence to Ning Quan, nquan@health.fau.edu
}

Received 18 October 2019; Accepted 18 October 2019

Copyright ( 2019 Ning Quan. This is an open access article distributed under the terms of the Creative Commons Attribution License, which permits unrestricted use, distribution, and reproduction in any medium, provided the original work is properly cited.

Psychoneuroimmunology (PNI) was a term first coined in 1975 by Robert Ader and Nicholas Cohen, the founding fathers of the Psychoneuroimmunology Research Society (PNIRS). This discipline draws from the ancient wisdom that body and mind are inseparable, germinates from the modern revelation that psychological, neurological, and immunological functions are interwoven by overlapping molecular and cellular processes, and is evolving to reshape our understanding of previously isolated biological systems into networks of interdependent, cooperative modules that connect to form a whole.

The spirit of PNI has guided medical practice since antiquity. The ancient Chinese classic "Plain Inquiries", dated about $250 \mathrm{BCE}$, stated "problems of the heart arise from over-indulged joy, anger injures the liver, worry damages the lung, longing unsettles the spleen, and fear hollows the kidney". While the insight in this text that different emotional states have profound impact on the health of specific organs was astonishingly prescient from so long ago, the precise connections stated were certainly inaccurate. This example highlights the two key challenges in PNI research since its inception, viz., the identification of connections among widely disparate fields of psychology, neurobiology, and immunology, and the elucidation of the precise conditions for these connections such that physiological and pathophysiological phenomena can be explained and predicted.

A modern example here is the afferent neuroimmune communication pathway. Significant progress has been made in the last four decades demonstrating activation of peripheral immune system results in the engagement of the central nervous system (CNS) via five pathways: (1) activation of the circumventricular organs of the brain, (2) activation of the brain endothelial production of signaling molecules, (3) activation of blood brain barrier transport systems, (4) activation of sensory afferent of the peripheral nervous system, and (5) activation of the CNS via recruited peripheral leukocytes. Most researchers have settled on the notion that these pathways are redundant, but no single neuroimmune pathway is the only path for relaying peripheral immune signal to the brain. The implications of these five pathways, however, are drastically different. Thus, only the fourth pathway allows the brain to gather local immune information and the fifth pathway may be a sign of inappropriate neuroimmune communication as the recruitment of inflamed leukocytes into the sensitive CNS tissue without the inciting agent does not make physiological sense. The conditions that determine the selection of a specific neuroimmune pathway remains to be defined.

Similarly, recent studies have shown cells of the immune system exert powerful influence on cognitive and affective functions of the brain. Considering neurons related to cognition and emotion communicate with each other by action potential in the time frame of fractions of second in hardwired neurocircuits, whereas immune cells are mobile, move slowly, and are generally rare in the brain, the way immune cells impinge onto neuro-circuits to produce neuroimmune effects is far from being understood. The goals of this journal, therefore, are to encourage researchers to publish their discoveries of connections in the supra-system of psychoneuroimmunology and their critical evaluations of the conditions under which these systems influence each other to produce meaningful physiological and pathophysiological outcomes. We believe only with such comprehensive understanding, PNI-based medical practice can be truly advanced.

Ning Quan Editor-in-Chief 